\title{
Earthquake analysis of thick plates resting on elastic foundation with second order finite element
}

\author{
Y.I. Özdemir* \\ Department of Civil Engineering, Karadeniz Technical University, 61080 Trabzon, Turkey
}

\begin{abstract}
The purpose of this paper is to study parametric earthquake analysis of thick plates resting on Winkler foundation using Mindlin's theory, to determine the effects of the thickness/span ratio, the aspect ratio and the boundary conditions on the linear responses of thick plates subjected to earthquake excitations. In the analysis, finite element method is used for spatial integration and the Newmark- $\beta$ method is used for the time integration. Finite element formulation of the equations of the thick plate theory is derived by second order displacement shape functions. A computer program using finite element method is coded in $\mathrm{C}++$ to analyze the plates simply supported and clamped along all four edges. In the analysis, 8-noded finite element is used. Graphs are presented that should help engineers in the design of thick plates subjected to earthquake excitations. It is concluded that 8-noded finite element can be effectively used in the earthquake analysis of thick plates. It is also concluded that, in general, the changes in the thickness/span ratio are more effective on the maximum responses considered in this study than the changes in the aspect ratio.
\end{abstract}

\section{Keywords}

Earthquake Analysis; Thick Plate; Mindlin's Theory; 8-Noded Finite Element; Winkler foundation

Received: 29 March 2018; Accepted: 21 June 2018

ISSN: 2630-5763 (online) C 2018 Golden Light Publishing® All rights reserved.

\section{Introduction}

The plates resting on elastic foundation is one of the most popular topics for the last decade in many engineering applications. Winkler model, Pasternak model, Hetenyi model, Vlasov and Leont'ev model are the models used by the researchers to calculate the soil effects on the plate.

Winkler model is used as a set of uncorrelated elastic springs attached to each node of the plate [1]. In this method, the deflections are only related with the load on the plate. The deflection of neighbouring points of the foundation is independent of each other. Hetenyi [2] proposed a two-parameter model, Pasternak model takes in to account the effects of shear interaction among joining points in the foundation [3]. Vlasov and Leont'ev [4] related the solution with a $\gamma$ parameter which is calculated with soil material and thickness of the soil. Winkler model is the simplest model for elastic foundations so the author used in this study this model.

The dynamic behavior of thick elastic plates has been investigated by many researchers [5-7]. Omurtag and Kadioğlu [8] are studied free vibration analysis of orthotropic plates resting on Pasternak foundation by mixed finite element formulation, Ayvaz and Oğuzhan [9] are analysis free vibration of thick plates resting on Vlasov elastic foundation.

\footnotetext{
Corresponding author

E-mail: yozdemir@,ktu.edu.tr
} 
The purpose of this paper is to study parametric earthquake analysis of thick plates resting on Winkler foundation, to determine the effects of the thickness/span ratio, the aspect ratio and boundary condition on the linear responses of the thick plates subjected to earthquake excitations. A computer program using finite element method is coded in $\mathrm{C}++$ to analyses the plates simply supported and clamped along all four edges. In the program, the finite element method is used for spatial integration and the Newmark- $\beta$ method is used for the time integration. Finite element formulation of the equations of the thick plate theory is derived by using second order displacement shape functions. In the analysis, 8-noded finite element is used to construct the stiffness and mass matrices.

\section{Mathematical model}

The governing equation for a flexural plate (Fig. 1) subjected to an earthquake excitation without damping can be given as [10]

$$
[M]\{\ddot{w}\}+[K]\{w\}=[F]=-[M]\left\{\ddot{u}_{g}\right\}
$$

where $[K]$ and $[M]$ are the stiffness matrix and the mass matrix of the plate, respectively, $w$ and $\ddot{w}$ are the lateral displacement and the second derivative of the lateral displacement of the plate with respect to time, respectively, $\ddot{u}_{g}$ is the earthquake acceleration.

In order to do forced vibration analysis of a plate, the stiffness, $[K]$, mass matrices, $[M]$, and equivalent nodal loads vector, $[F]$, of the plate should be constructed. The evaluation of these matrices is given in the following sections.

The total strain energy of plate-soil-structure system (see Fig. 1) can be written as;

$$
\Pi=\Pi_{p}+\Pi_{s}+V
$$

where $\Pi_{p}$ is the strain energy in the plate as

$$
\begin{aligned}
& \Pi_{p}=\frac{1}{2} \int_{A}\left(-\frac{\partial \phi_{x}}{\partial x} \frac{\partial \phi_{y}}{\partial y}-\frac{\partial \phi_{x}}{\partial y}+\frac{\partial \phi_{y}}{\partial x}\right)^{T} E_{\kappa}\left(-\frac{\partial \phi_{x}}{\partial x} \quad \frac{\partial \phi_{y}}{\partial y}-\frac{\partial \phi_{x}}{\partial y}+\frac{\partial \phi_{y}}{\partial x}\right) d_{A} \\
& +\frac{k}{2} \int_{A}\left(-\phi_{x}+\frac{\partial w}{\partial x} \phi_{y}+\frac{\partial w}{\partial y}\right)^{T} E_{\gamma}\left(-\phi_{x}+\frac{\partial w}{\partial x} \quad \phi_{y}+\frac{\partial w}{\partial y}\right) d_{A}
\end{aligned}
$$
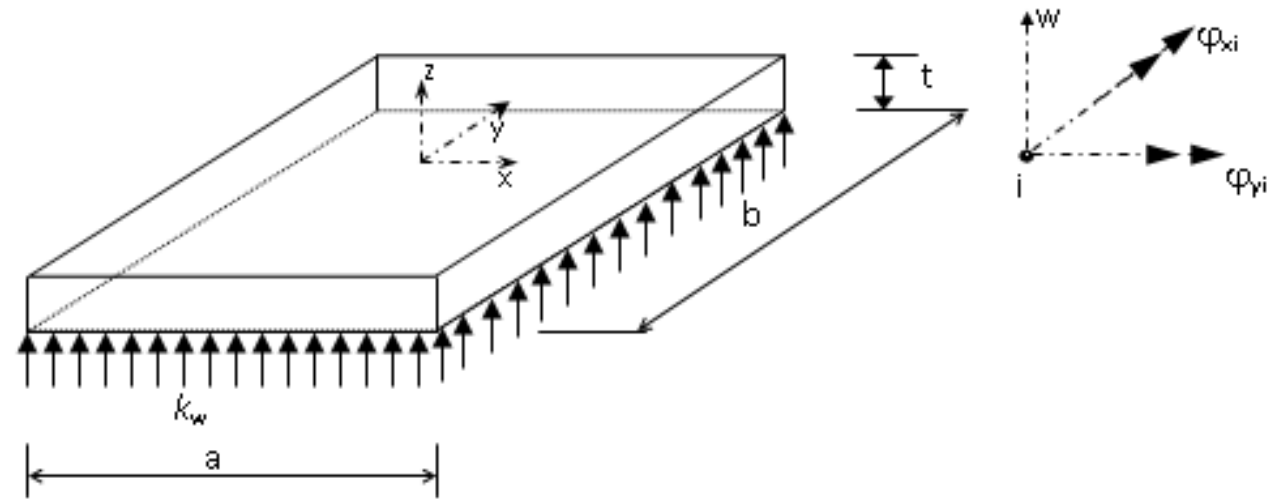

Fig. 1. The sample plate used in this study 
and $\Pi_{s}$ is the strain energy stored in the soil as

$$
\Pi_{s}=\frac{1}{2} \int_{0}^{H} \int_{-\infty}^{\infty} \int_{-\infty}^{\infty} \sigma_{i j} \varepsilon_{i j}
$$

and $V$ is the potential energy of the earthquake loading

$$
\begin{aligned}
& V=-\int_{A} \bar{q} w d_{A} \\
& \Pi=\frac{1}{2} \int_{A}\left(-\frac{\partial \phi_{x}}{\partial x} \frac{\partial \phi_{y}}{\partial y}-\frac{\partial \phi_{x}}{\partial y}+\frac{\partial \phi_{y}}{\partial x}\right)^{T} E_{\kappa}\left(-\frac{\partial \phi_{x}}{\partial x} \quad \frac{\partial \phi_{y}}{\partial y}-\frac{\partial \phi_{x}}{\partial y}+\frac{\partial \phi_{y}}{\partial x}\right) d_{A} \\
& +\frac{k}{2} \int_{A}\left(-\phi_{x}+\frac{\partial w}{\partial x} \phi_{y}+\frac{\partial w}{\partial y}\right)^{T} E_{\gamma}\left(-\phi_{x}+\frac{\partial w}{\partial x} \quad \phi_{y}+\frac{\partial w}{\partial y}\right) d_{A}+\frac{1}{2} \int_{A}\left(w_{x, y}\right)^{T} K\left(w_{x, y}\right) d_{A}
\end{aligned}
$$

In this equation, $E_{\kappa}$ and $E_{\gamma}$ are the elasticity matrices that will be given in Eq. (14), $\bar{q}$ shows earthquake loading.

\subsection{Evaluation of the stiffness matrix}

The total strain energy of the plate-soil system
In this equation, the first and second part gives the conventional element stiffness matrix of the plate, $k_{p}^{e}$, differentiation of the third integral with respect to the nodal parameters yields a matrix $k_{w}^{e}$, which accounts for the axial strain effect in the soil. Thus the total energy of the plate-soil system can be written as;

$$
\Pi=\frac{1}{2}\left\{w_{e}\right\}^{T}\left(\left[k_{p}^{e}\right]+\left[k_{w}^{e}\right]\right)\left\{w_{e}\right\} d_{A}
$$

where

$$
\left\{w_{e}\right\}=\left[\begin{array}{lllllll}
w_{1} & \phi_{y 1} & \phi_{x 1} & \ldots & w_{n} & \phi_{y n} & \phi_{x n}
\end{array}\right]^{T}
$$

Assuming that, in the plate of Fig. 1, $u$ and $v$ are proportional to $\mathrm{z}$ and $w$ is the independent of $z$ [11], one can write the plate displacement at an arbitrary $x, y, z$ in terms of the two slopes and a displacement as follows:

$$
\{w, v, u\}=\left\{w_{0}(x, y, t), z \varphi_{y}(x, y, t),-z \varphi_{x}(x, y, t)\right\}
$$

where $w_{0}$ is average displacement of the plate, and $\varphi_{x}$ and $\varphi_{y}$ are the bending slopes in the $y$ - and $x$ directions, respectively.

The nodal displacements for 8-noded quadrilateral serendipity element (MT8) (see Fig. 2) can be written as follows:
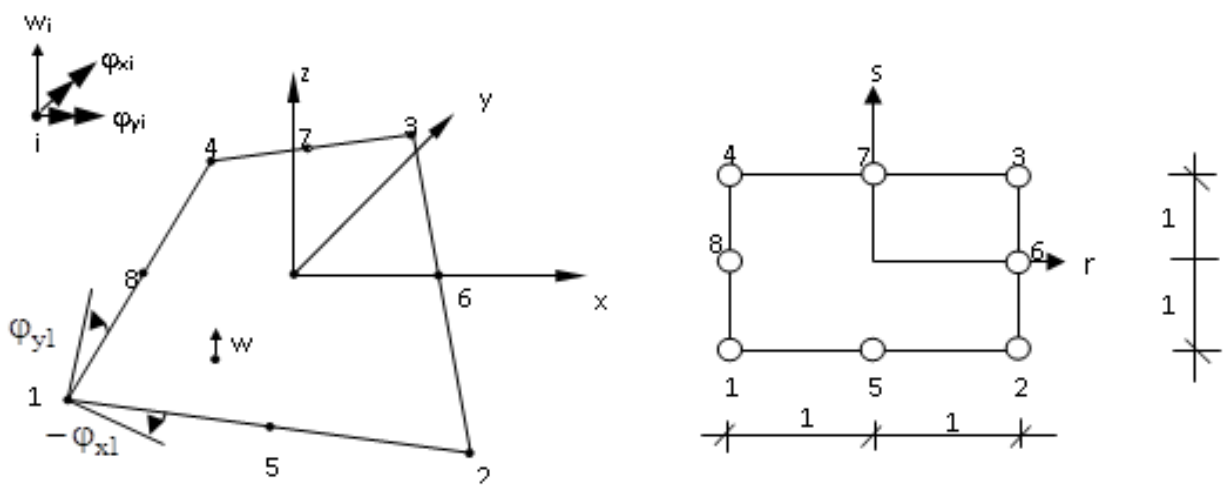

Fig. 2. 8-noded quadrilateral finite element used in this study [12] 
$u=z \varphi_{x}=-z \sum_{i=1}^{8} h_{i} \varphi_{x i}, \quad v=z \varphi_{y}=z \sum_{i=1}^{8} h_{i} \varphi_{y i}$,

$w=\sum_{i=1}^{8} h_{i} w_{i}$

The displacement function chosen for this element is;

$w=c_{1}+c_{2} r+c_{3} s+c_{4} r^{2}+c_{5} r s+c_{6} s^{2}+c_{7} r^{2} s+c_{8} r s^{2}$

From this assumption, it is possible to derive the displacement shape function to be;

$h=\left[h_{1}, h_{2}, h_{3}, h_{4}, h_{5}, h_{6}, h_{7}, h_{8}\right]$

Then, the strain-displacement matrix $[B]$ for this element can be written as follows Cook et al. [13]:

$[B]=\left[\begin{array}{cccc}0 & 0 & -\frac{\partial h_{i}}{\partial x} & \cdots \\ 0 & \frac{\partial h_{i}}{\partial y} & 0 & \ldots \\ 0 & \frac{\partial h_{i}}{\partial x} & -\frac{\partial h_{i}}{\partial y} & \cdots \\ \frac{\partial h_{i}}{\partial x} & 0 & -h_{i} & \cdots \\ \frac{\partial h_{i}}{\partial y} & h_{i} & 0 & \cdots\end{array}\right]_{5 \times 24}$

where $i=1,2, \ldots, 8$.

The stiffness matrix for MT8 element can be obtained by the following equation [13] as

$k_{p}=\int_{V} B^{T} E B d V=\int_{V}\left[z \bar{B}_{k}^{T} \quad B_{\gamma}^{T}\right]\left[\begin{array}{cc}E_{k} & 0 \\ 0 & E_{\gamma}\end{array}\right]\left[\begin{array}{c}z \bar{B}_{k}^{T} \\ B_{\gamma}^{T}\end{array}\right] d V$

$k_{p}=\int_{V}\left(z^{2} \bar{B}_{k}^{T} E_{k} \bar{B}_{k}\right)+\left(\bar{B}_{\gamma}^{T} E_{\gamma} \bar{B}_{\gamma}\right) d V$.

Integration of Eq. (14) through the thickness yields

$k_{p}=\int_{A}\left(\bar{B}_{k}^{T} \bar{E}_{k} \bar{B}_{k}+\bar{B}_{\gamma}^{T} \bar{E}_{\gamma} \bar{B}_{\gamma}\right) d A$

where the first term concerns with the bending and the second term concerns with the shear effects of the thick plate. Thus,
$k_{p}=\int_{A} \bar{B}^{T} \bar{E} \bar{B} d A=\int_{-1}^{1} \int_{-1}^{1} \bar{B}^{T} \bar{E} \bar{B}|J| d r d s$

which must be evaluated numerically [12]

\subsection{Foundation formulation}

As explained before, Winkler model is the simplest model for the plates resting on elastic foundation. In this model, all the deflections on the plate are due to the load on it. The foundation is represented with a set of uncorrelated elastic springs. So, in the analysis, the stiffness of these springs is calculated and is added to the element stiffness matrix. The stiffness matrices for the Winkler foundation can be derived by;

$k_{w}=k \int_{-1}^{1} \int_{-1}^{1}[h]^{T}[h]|J| d r d s$

where $k$ is the elastic foundation modulus.

After calculating all element stiffness matrices, global stiffness matrix can be assembled as;

$[K]=\sum_{i=1}^{p_{e}}\left(\left[k_{p}\right]+\left[k_{w}\right]\right)$

where $p_{e}$ is the node number.

\subsection{Evaluation of the mass matrix}

The formula for the consistent mass matrix of the plate may be written as

$M=\int_{\Omega} H_{i}^{T} \mu H_{i} d \Omega$

In this equation, $\mu$ is the mass density matrix of the form [10]

$\mu=\left[\begin{array}{ccc}m_{1} & 0 & 0 \\ 0 & m_{2} & 0 \\ 0 & 0 & m_{3}\end{array}\right]$

where $m_{1}=\rho_{p} t, m_{2}=m_{3}=\rho_{p} t^{3} / 12$, and $\rho_{p}$ is the mass densities of the plate and $H_{i}$ can be written as follows

$H_{i}=\left[d h_{i} / d x \quad d h_{i} / d y \quad h_{i}\right] \quad i=1,2, \ldots, 8$ 
It should be noted that the rotation inertia terms are not taken into account. By assembling the element mass matrices obtained, the system mass matrix is obtained.

\subsection{Evaluation of equivalent nodal loads vector}

Equivalent nodal loads, $[F]$, can be obtained by the following equation.

$$
F=\int H_{i}^{T} \bar{q} d \Omega
$$

In this equation, $H_{i}$ can be obtained by Eq. (21), and $\bar{q}$ denotes

$\bar{q}=-[M]\left\{\ddot{u}_{g}\right\}$

It should be noted that, in this study, the program, MATLAB ${ }^{\circledR}$ [14], is used for the eigenvalue solution of Eq. (1). It should also be noted that, the Newmark- $\beta$ method is used for the time integration of Eq. (2) by using the average acceleration method.

\section{Numerical examples}

In the light of the results given in the references $[15,16]$, the aspect ratios, $b / a$, of the plate are taken to be 1, 2.0, and 3.0. The thickness/span ratios, $t / a$, are taken as $0.05,0.1,0.2$, and 0.3 for each aspect ratio. The shorter span length of the plate is kept constant to be $3 \mathrm{~m}$. The mass density, Poisson's ratio, and the modulus of elasticity of the plate are taken to be $2.5 \mathrm{kNs}^{2} / \mathrm{m}^{2}, 0.2$, and $2.7 \times 10^{7} \mathrm{kN} / \mathrm{m}^{2}$. Shear factor $k$ is taken to be $5 / 6$. The subgrade reaction modulus of the Winkler-type foundation is taken to be $5000 \mathrm{kN} / \mathrm{m}^{3}$.

In the time history analysis to obtain the response of each plate the first 10 s of Vertical component of the January 17, 1995 Kobe earthquake in Japan is used. Duration of this earthquake is 20 s, but the peak value of the record occurred in the first 10s of the earthquake (Fig. 3). For the sake of accuracy in the results, rather than starting with a set of a finite element mesh size and time increment, the mesh size and time increment required to obtain the desired accuracy were determined before presenting any results This analysis was performed separately for the mesh size and time increment. It was concluded that the results have acceptable error when equally spaced $8 \times 8$ mesh sizes are used for a $3 \times 3 \mathrm{~m}$ plate even if it is a thin plate, if the $0.01 \mathrm{~s}$ time increment is used. Length of the elements in the $\mathrm{x}$ and $\mathrm{y}$ directions are kept constant for different aspect ratios as in the case of square plate.

One of the purposes of this paper was to determine the time histories of the displacements and the bending moments at different points of the thick plates subjected to earthquake excitations, but presentation of all of the time histories would take up excessive space. Hence, only the absolute maximum displacements and bending moments for different thickness/span ratio and aspect ratio are presented after two time histories are given. This simplification of presenting only the maximum responses is supported by the fact that the maximum values of these quantities are the most important ones for design. These results are presented in graphical rather than in tabular form.

The absolute maximum displacements of the thick plates for different aspect ratios, and thickness/span ratios are given in Fig. 4 for the thick plates simply supported along all four edges.

As seen from Fig. 4, the absolute maximum displacements of the thick plates increase with increasing aspect ratio for a constant $t / a$ ratio. The same displacements decrease with increasing t/a ratio for a constant $b / a$ ratio. As also seen from these figures, the decrease in the absolute maximum displacement for a constant $b / a$ ratio increases with increasing $b / a$ ratio. The curves for a constant value of the aspect ratio, b/a are fairly getting closer to each other as the value of $t / a$ increases. This shows that the curves of the absolute maximum displacements will almost coincide with each other when the value of the thickness/span ratio, $t / a$, increases more. In other words, the increase in the thickness/span ratio will not affect the absolute maximum displacements after a determined value of $t / a$. 


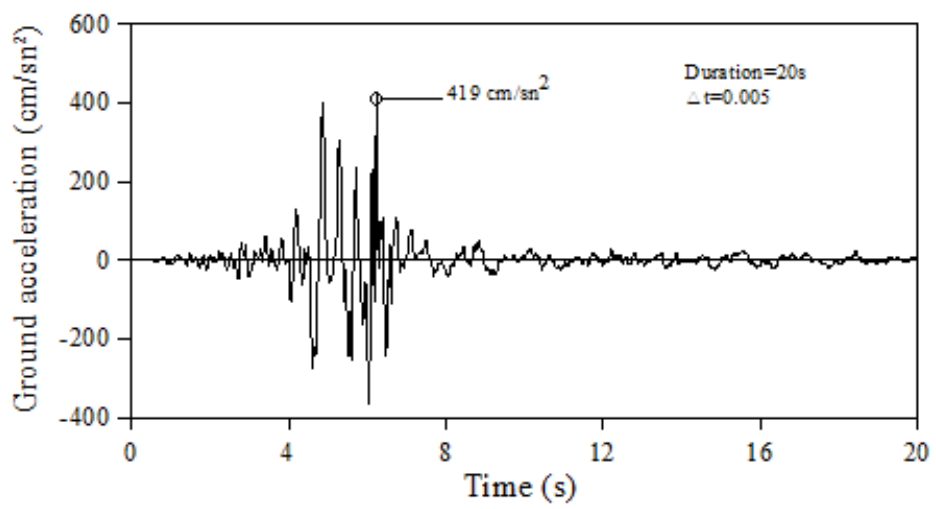

Fig. 3. Vertical component of the January 17, 1995 Kobe earthquake in Japan

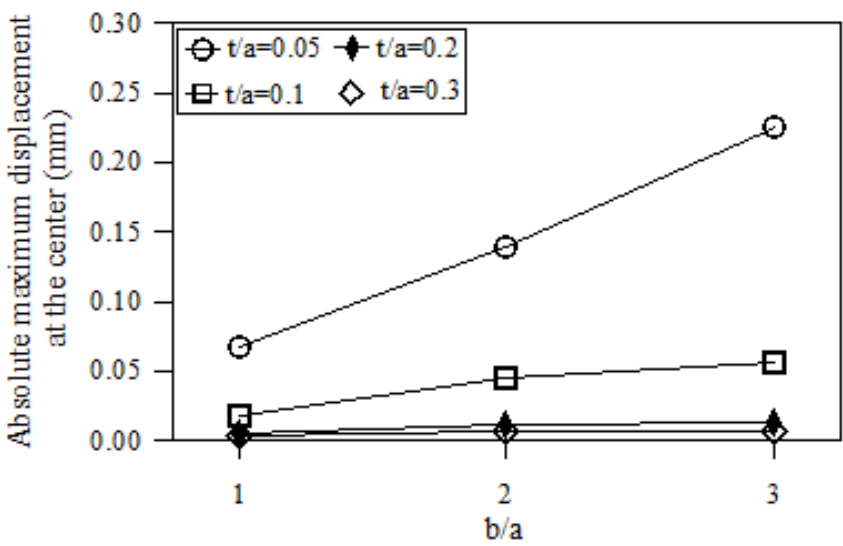

Fig. 4. Absolute maximum displacement of the reinforced concrete thick simply supported plates resting on Winkler foundation for different aspect ratios and thickness/span ratios.

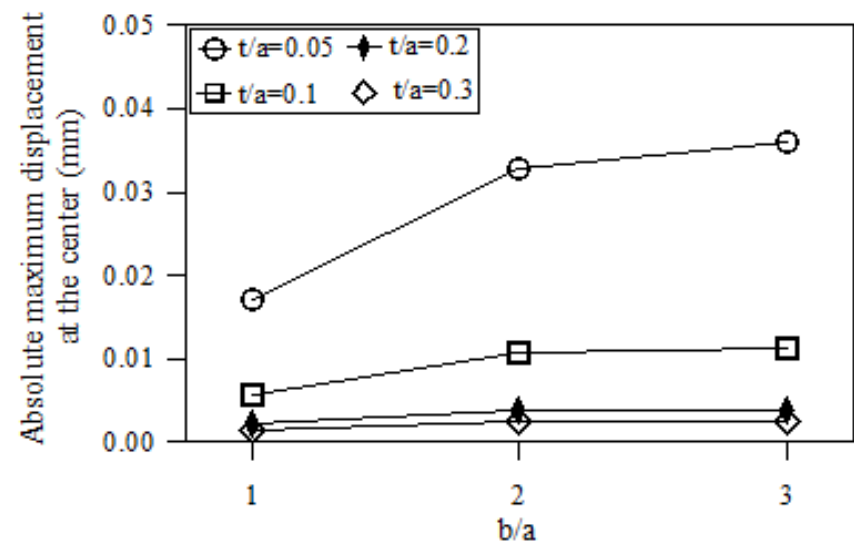

Fig. 5. Absolute maximum displacement of the reinforced concrete thick clamped plates resting on Winkler foundation for different aspect ratios and thickness/span ratios.

The absolute maximum displacements of the thick plates for different aspect ratios, and thickness/span ratios are given in Fig. 5 for the thick plates clamped along all four edges.
As seen from Fig. 5, the absolute maximum displacements of the thick plates increase with increasing aspect ratio for a constant $t / a$ ratio. The same displacements decrease with increasing $t / a$ 
ratio for a constant $b / a$ ratio. As also seen from these figures, the decrease in the absolute maximum displacement for a constant $b / a$ ratio increases with increasing $b / a$ ratio. The curves for a constant value of the aspect ratio, b/a are fairly getting closer to each other as the value of $t / a$ increases. This shows that the curves of the absolute maximum displacements will almost coincide with each other when the value of the thickness/span ratio, $t / a$, increases more. In other words, the increase in the thickness/span ratio will not affect the absolute maximum displacements after a determined value of $t / a$.

The absolute maximum bending moments $M_{x}$ at the center of the thick plates for different aspect ratios and thickness/span ratios are given in Fig. 6 for the thick simply supported plates. As seen from Fig. 6, the absolute maximum bending moment, $M_{x}$, at the center of the thick simply supported plates increases with increasing aspect ratio and thickness/span ratio. The increases in the absolute maximum bending moment, $M_{x}$, increase with increasing aspect and thickness/span ratios. This is understandable that increasing the aspect ratio makes the plate stiffer in the short span, the $\mathrm{x}$ axis, direction. As also seen from this figure, in general, the effects of the changes in the aspect ratios on the absolute maximum bending moment, $M_{x}$, are larger than the changes in the thickness/span ratios.

The absolute maximum bending moments $M_{x}$ at the center of the thick plates for different aspect ratios and thickness/span ratios are given in Fig. 7 for the thick clamped plates.

As seen from Fig. 7, the absolute maximum bending moment, $M_{x}$, at the center of the thick clamped plates, as in the case of the absolute maximum bending moment, $M_{x}$, at the center of the thick and thin simply supported plates, increases with increasing aspect ratio and thickness/span ratio. The increases in the absolute maximum bending moment, $M_{x}$, increase with increasing aspect and thickness/span ratios. This is also understandable that increasing the aspect ratio makes the plate stiffer in the short span, the $x$-axis, direction. As also seen from this figure, in general, the effects of the changes in the aspect ratios on the absolute maximum bending moment, $M_{x}$, are larger than the changes in the thickness/span ratios.

The absolute maximum bending moments $M_{y}$ at the center of the thick plates for different aspect ratios and thickness/span ratios are given in Fig. 8 for the thick simply supported plates.

As seen from Fig. 8, the absolute maximum bending moment, $M_{y}$, at the center of the thick simply supported plates decreases with increasing aspect ratio and increases with increasing thickness/span ratio. The decrease in the absolute maximum bending moment, $M_{y}$, increase with increasing aspect ratio. The increase in the absolute maximum bending moment, $M_{y}$, increases with increasing thickness/span ratios. This is understandable that increasing the aspect ratio makes the thick plates more flexible in the long span, the y axis, direction. As also seen from this figure, in general, the effects of the changes in the thickness/span ratios on the absolute maximum bending moment, $M_{y}$, are larger than the changes in the aspect ratios.

The absolute maximum bending moments $M_{y}$ at the center of the thick plates for different aspect ratios and thickness/span ratios are given in Fig. 9 for the thick clamped plates.

As seen from Fig. 9, the absolute maximum bending moment, $M_{y}$, at the center of the thick clamped plates, as in the case of the absolute maximum bending moment, $M_{y}$, at the center of the thick simply supported plates, decreases with increasing aspect ratio and increases with increasing thickness/span ratio. The decrease in the absolute maximum bending moment, $M_{y}$, increase with increasing aspect ratio. The increase in the absolute maximum bending moment, $M_{y}$, increases with increasing thickness/span ratios. This is also understandable that increasing the aspect ratio makes the thick plates more flexible in the long span, the $y$-axis, direction. As also seen from this figure, in general, the effects of the changes in the thickness/span ratios on the absolute maximum bending moment, $M_{y}$, are larger than the changes in the aspect ratios. 


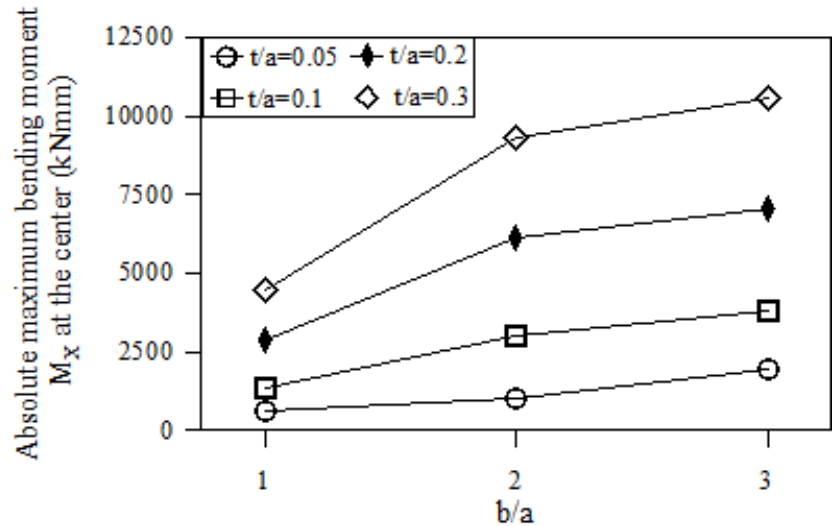

Fig. 6. Absolute maximum bending moment $M_{x}$ at the center of the reinforced concrete thick simply supported plates resting on Winkler foundation for different aspect ratios and thickness/span ratios.

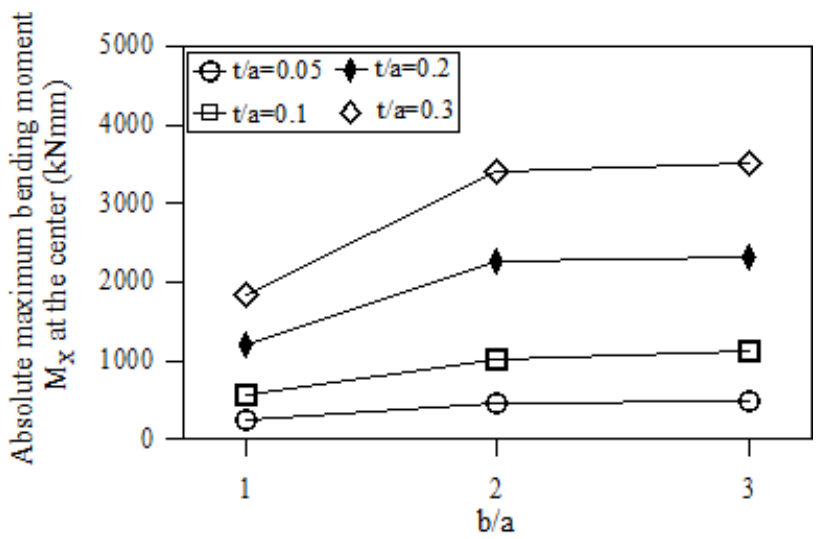

Fig. 7. Absolute maximum bending moment $M_{x}$ at the center of the reinforced concrete thick clamped plates resting on Winkler foundation for different aspect ratios and thickness/span ratios.

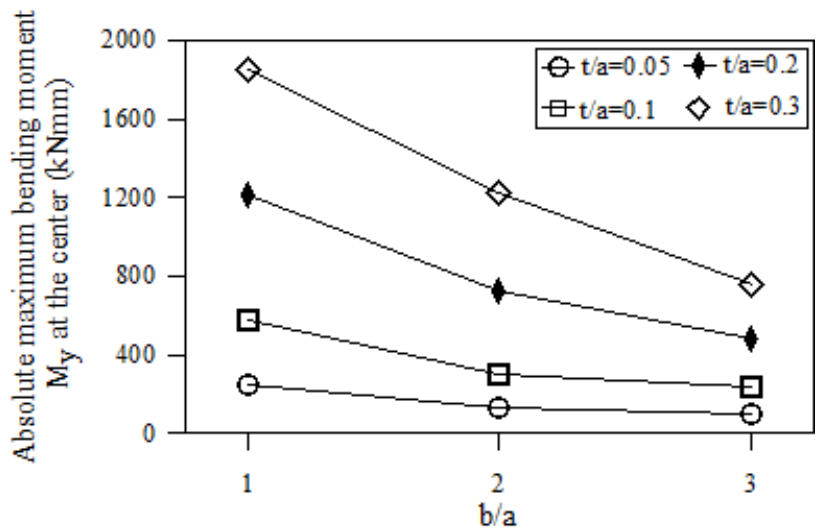

Fig. 8. Absolute maximum bending moment $M_{y}$ at the center of the reinforced concrete thick simply supported plates resting on Winkler foundation for different aspect ratios and thickness/span ratios. 


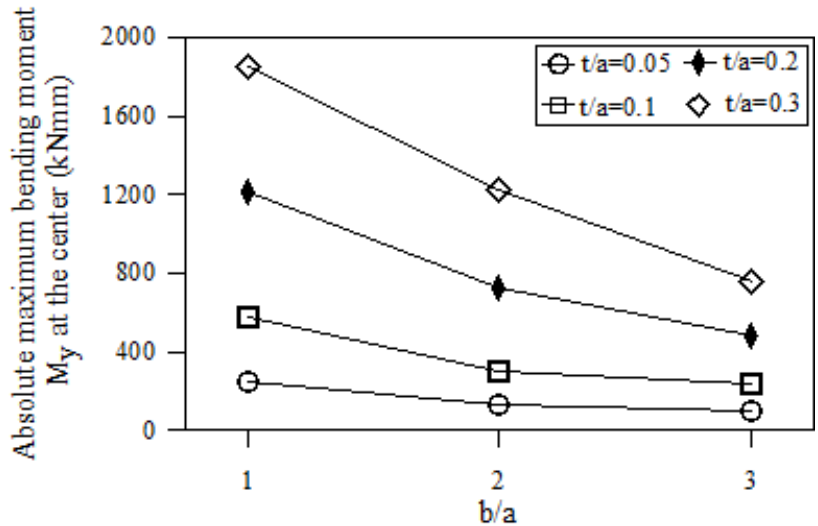

Fig. 9. Absolute maximum bending moment $M_{y}$ at the center of the reinforced concrete thick clamped plates resting on Winkler foundation for different aspect ratios and thickness/span ratios.

\section{Conclusions}

The purpose of this paper is to study shear lockingfree parametric earthquake analysis of thick plates resting on Winkler foundation, to determine the effects of the thickness/span ratio, the aspect ratio and boundary condition on the linear responses of the thick plates resting on Winkler foundation subjected to earthquake excitations. It is concluded that 8-noded finite element can be effectively used in the earthquake analysis of thick plates resting on elastic foundation. The coded program can be effectively used in the earthquake analyses of any thick plates resting on elastic foundation. It is also concluded that, in general, the changes in the thickness/span ratio are more effective on the maximum responses considered in this study than the changes in the aspect ratio.

In order to generalize the results obtained in this study, the responses of the different thick plates resting on Winkler foundation subjected to different earthquake excitations should be evaluated all together. Therefore, the curves presented herein can help the designer to anticipate the effects of the thickness/span ratio, the aspect ratio, and boundary condition on the earthquake response of a thick plate resting on Winkler foundation.

The following conclusions can also be drawn from the results obtained in this study.
- The absolute maximum displacements of the thick plates increase as the aspect ratio increases for a constant $t / a$ ratio. The same displacements decrease as the $t / a$ ratio increases for a constant $b / a$ ratio.

- The changes in the aspect ratios are generally less effective on the absolute maximum displacement than the changes in the thickness/span ratios.

- The absolute maximum bending moment, $M_{x}$, at the center of the thick simply supported plates resting on Winkler foundation increases as the aspect ratio and thickness/span ratio increase.

- The changes in the aspect ratios are generally more effective on the absolute maximum bending moment, $M_{x}$, of the thick simply supported plates than the changes in the thickness/span ratios.

- The absolute maximum bending moment, $M_{x}$, at the center of the thick clamped plates resting on Winkler foundation increases with increasing aspect ratio and thickness/span ratio.

- The changes in the aspect ratios are generally more effective on the absolute maximum bending moment, $M_{x}$, of the thick clamped plates resting on Winkler foundation than the changes in the thickness/span ratios. 
- The absolute maximum bending moment, $M_{y}$, at the center of the thick simply supported plates resting on Winkler foundation decreases as the aspect ratio increases and increases as the thickness/span ratio increases.

- The changes in the thickness/span ratios are generally more effective on the absolute maximum bending moment, $M_{y}$, of the thick simply supported plates resting on Winkler foundation larger than the changes in the aspect ratios.

- The absolute maximum bending moment, $M_{y}$, at the center of the thick clamped plates resting on Winkler foundation decreases with increasing aspect ratio and increases with increasing thickness/span ratio.

- The changes in the thickness/span ratios are generally more effective on the absolute maximum bending moment, $M_{y}$, of the thick clamped plates resting on Winkler foundation than the changes in the aspect ratios.

- In general, degrees of decreases and increases depend on the changes in the aspect and thickness/span ratios, and the changes in the thickness/span ratio are more effective on the maximum responses considered in this study than the changes in the aspect ratio.

\section{References}

[1] Winkler E. Theory of Elasticity and Strength. Dominicus, Pague, Czechoslovakia, 1867.

[2] Hetenyi M (1950) A general solution for the bending of beams on an elastic foundation of arbitrary continuity. Journal of Applied Physics 21: 55-58.

[3] Pasternak PL (1954) New method of calculation for flexible substructures on two-parameter elastic foundation. Gosudarstvennoe Izdatelstvo Literatura po Stroitelstvo i Arhitektura 1-56 (in Russian).

[4] Vlasov VZ, Leont'ev NN. Beam, Plates and Shells on Elastic Foundations. GIFML, Moskow, 1989.
[5] Tahouneh V (2014) Free vibration analysis of thick CGFR annular sector plates resting on elastic foundations. Structural Engineering Mechanics 50(6): 773-796.

[6] Benferhat R, Daouadji TH, Mansour MS, Hadji L (2016) Effect of porosity on the bending and free vibration response of functionally graded plates resting on Winkler-Pasternak foundations. Earthquakes \& Structures 10(6): 1429-1449.

[7] Senjanovic I, Tomic M, Hadzic N, Vladimir N (2017) Dynamic finite element formulations for moderately thick plate vibrations based on the modified Mindlin theory. Engineering. Structures 136: 100-113.

[8] Omurtag MH, Kadıoğlu F (1998) Free vibration analysis of orthotropic plates resting on Pasternak foundation by mixed finite element formulation. Computers \& Structures 67: 253265.

[9] Ayvaz Y, Oğuzhan CB (2008) Free vibration analysis of plates resting on elastic foundations using modified Vlasov model. Structural Engineering Mechanics 28(6): 635-658.

[10] Tedesco JW, McDougal WG, Ross CA. Structural Dynamics. Addison Wesley Longman Inc., California, 1999.

[11] Mindlin RD (1951) Influence of rotatory inertia and shear on flexural motions of isotropic, elastic plates. Journal of Applied Mechanics 18: 31-38.

[12] Bathe KJ. Finite Element Procedures, Prentice Hall, Upper Saddle River, New Jersey, 1996.

[13] Cook RD, Malkus DS, Michael EP. Concepts and Applications of Finite Element Analysis. John Wiley \& Sons, Inc., Canada, 1989.

[14] Özdemir YI, Bekiroğlu S, Ayvaz Y (2007) Shear locking-free analysis of thick plates using Mindlin's theory, Structural Engineering Mechanics, 27(3): 311-331.

[15] Özdemir YI (2012) Development of a higher order finite element on a Winkler foundation. Finite Element. Analysis and Design 48: 14001408 . 